oxalic acid content of certain foods and recommended daily allowances for specific nutrients.

The very fact that we are islanders surrounded by the sea, the spray of which falls on and effects our lands and food products, brings home to us the problem of solving the ever-present topic of variety in diet. This is especially difficult with a patient who is ill and feels that the illness alone is enough to bear without the burden of an unpalatable diet.

This revised edition has been eagerly awaited and reader will not be disappointed.

\section{MEDICAL BOTANY}

By Alexander Nelson, Ph.D., D.Sc., F.R.S.E. Pp. $x i+544$, with 29 illustrations. Edinburgh: E. \& S. Livingstone. 195I. 303.

A previous book, 'Introductory Botany,' by the same author was written for medical, pharmaceutical, veterinary and pure science students. The present companion volume is a development of the subject beyond the student stage and is intended for the doctor, pharmacist, veterinary surgeon and dietician. It is conveniently divided into sections.

Section IA deals with the food values of vegetable food stuffs. After an account of the 'food structure' of plants in general there follows a more detailed classified account of the plant, carbohydrates, fats, proteins, mineral matter and vitamins. Some account of the effects on these food classes caused by storage, preserving and cooking brings the plant, as a food source, right up to the consumer stage. The treatment of the mineral and vitamin constituents in this section is particularly interesting. Section IB deals with the food stuffs in detail. Carbohydrates, for instance, are not merely described as starches, sugars, celluloses, etc., but the contribution of each part of the plant is considered separately. Fruits and seeds are classified into dry fruits, e.g. wheat, maize, barley, rice, oats, sorghums, millet, beans, peas, oilseeds and nuts, and into fleshy and juicy fruits such as apples, pears, pineapple, currants, gooseberries and many more. Each fruit or seed is given length of description in relation to its food value importance. Rice, for example, has a brief description of the whole plant, where it grows, the processing of the grain, the different types of rice grain, the composition of the grain, including its carbohydrate, fat, protein, mineral and vitamin content and, finally, the industrial uses of rice. This exhaustive treatment is applied in the same way to leaf, root and stem vegetables as well as to the food values of non-flowering organisms, mainly algae and fungi. This section contains some 270 pages and forms the major part of the book.

Section II devotes some roo pages to an account of vegetable drugs, poisonous, stimulants and vegetable products of pharmaceutical interest. This section is a general introduction to the pharmacology of vegetable products and touches on their therapeutic significance.

Section III is a brief summary of plants as the casual agents of disease. It indicates the effect of direct contact causing external, and inhaled dusts causing internal irritation. Diseases caused by ingesting plants or parts of plants and the pathogenic effects of fungi are briefly mentioned. Some few pages are devoted to allergy caused by plants and, finally, mention is made of the methods employed in identification of plants or parts of plants. There is an adequate index, a good point being the indication in Clarendon type as to where the more important treatment of any subject is to be found. Absence of a glossary would necessitate a good deal of dictionary work on the part of the lay reader, but even so he would find it an intriguing book. There is no bibliography, which is to be regretted, as the book itself stimulates a desire to go further into the subjects dealt with.

In a short review it is impossible to do this work justice. The reviewer can do no better than to quote the opinion expressed by one of the more able of his medical students who had just read the book and said that it is ' absolutely fascinating.'

W.H.W.

\section{CLINICAL PRACTICE IN INFECTIOUS DISEASES}

By E. H. R. Harries, M.D., F.R.C.P., and M. Mrtman, M.D., F.R.C.P. 4th Edition. Pp. $x+717$, with $5^{8}$ illustrations. Edinburgh: E. \& S. Livingstone. I95I. 30s.

Since its first publication in 1940, this book has been the standard textbook in infectious diseases, and although there have recently been several important new books in this field it is likely that, in its fourth edition, "Mitman and Harries" will remain the standard. It does not always read too easily, but description and information are almost always exact and detailed. The reviewer has tested this edition at various points and has found, in respect of recent advances, that it is well up-to-date. The chapter on poliomyelitis, however, is a little disappointing; one would have welcomed a fuller description of the bulbar forms and detailed discussion of the physiological and pathological factors involved in the correction of respiratory distress. There are only passing references to the Stevens Johnson syndrome-a not uncommon condition in fever hospital practice - and none at all to anthrax.

Several new illustrations appear in this edition and all are successful. The section on antibiotics has been brought up-to-date and there are many instances throughout the book-e.g. the blood picture in infectious mononucleosis and the laboratory diagnosis of smallpox - where brief but accurate additions have been made. An appendix on the Public Health Laboratory Service has been added and includes instructions on the specimens to be taken in different diseases. In the reviewer's opinion, this is of doubtful utility. The book was originally written for undergraduates but there is no doubt the authors have also thought of postgraduates and specialists, and this dual purpose occasionally leads to a lack of balance in the text. Nevertheless, it would be very seldom that even the specialist would refer to the book without getting the required information or the necessary reference. A.B.C. 